\title{
X-ray Studies on Rotational Transition of Molecular Chains in Polyethylene Crystal
}

\author{
Koh-ichi Iohara, Kiyohisa Imada, and Motowo Takayanagi \\ Department of Applied Chemistry, Faculty of Engineering, \\ Kyushu University, Fukuoka, Japan.
}

(Received March 10, 1972)

\begin{abstract}
By assuming the probability of the rotational transition of polyethylene molecules in the crystal by about $90^{\circ}$ around their chain axes, the average structure factor for X-ray diffraction was formulated. According to the formulas, the intensities of $h k l$ reflections with $h+k=$ even are independent of the transition, whereas those of reflections with $h+k=$ odd are decreased with the increasing fraction of rotated molecular chains. By analyzing the intensities of X-ray diffractions from single crystals and the bulk-crystallized sample at various temperatures, it was found that the molecular chains in such crystals showed an increasing tendency to be transformed from the normal orientation of the molecular zigzag plane to the abnormal orientation with increasing temperature above $0^{\circ} \mathrm{C}$. The latter fraction amounted to $5 \%$ in the crystal at $120^{\circ} \mathrm{C}$.

KEY WORDS X-ray / Rotational Transition / Structure Factor / Crystal Defect / Molecular Motion / Polyethylene
\end{abstract}

Molecular motions in polyethylene crystals have been investigated by using various methods, including measurements of mechanical properties $^{1,2}$, nuclear magnetic resonance $(\mathrm{NMR})^{3,4}$ and X-ray diffraction ${ }^{5-11}$.

The $a$ axis of polyethylene crystals showed a tendency of larger thermal expansion at temperatures above $0^{\circ} \mathrm{C}$, which suggests that the lattice vibrations can no longer remain harmonic above this temperature ${ }^{5}$. This indication was also supported by the temperature factor determined by $\mathrm{X}$-ray diffraction intensity ${ }^{6,7,12}$. $\mathrm{We}^{1,2}$ have observed the mechanical $\alpha$-absorption associated with the crystalline region in the temperature region between $0^{\circ} \mathrm{C}$ and the melting temperature in the mechanical relaxation curves, and proposed mechanisms for the dispersion, that some anharmonic thermal motions of molecules take place in the interior of the crystal, giving rise to the mechanical loss caused by the intermolecular friction. This proposal was also supported by the results of the NMR measurements ${ }^{3,4}$ in which the narrowing of the broad component associated with the crystalline region occurred at the temperature corresponding to that of the crystalline absorption. Thus, it seems reasonable that anharmonic molecular motions with large amplitude are initiated at about $0^{\circ} \mathrm{C}$ in the crystal lattice and increase in intensity with increasing temperature. Several authors ${ }^{1-4,13-16}$ have considered that the representative modes of molecular motions of polyethylene chains are the rotational or torsional ones around their axes and the translational one along the same axes. These two modes with low normal vibrations are easily amplified and liable to become anharmonic at sufficiently high temperatures.

In order to clarify the process of rotational motion from an energetic viewpoint, several authors ${ }^{16-18,24}$ have calculated the intermolecular energy for rotation of polyethylene molecule around its chain axis in a fixed crystallographic environment. Such a calculation of the intermolecular energy predicts a second minimum when a molecule rotates by an angle of about $90^{\circ}$ around its chain axis from its normal orientation of the molecular zigzag plane.

In this study, we assumed the rotational transition of the molecular chain from the first minimum of the normal orientation to the second minimum of the abnormal one as one 
of the mechanisms of the molecular motions in the crystal above $0^{\circ} \mathrm{C}$. By formulating the average structure factor for X-ray diffraction intensity by taking into account the rotational transition of the molecular chains, we will evaluate the probability of the rotational transition by using the observed intensities of X-ray diffraction to depict the change of state of polyethylene crystals at elevated temperatures.

\section{THEORETICAL}

Figure 1 shows the variation of the intermolecular energy by rotation of a molecular chain around its chain axis calculated by Wada and Hayakawa ${ }^{16}$. The angle $\theta$ denotes the one formed between the molecular zigzag plane and the $b$ axis. The angle (about $45^{\circ}$ ) giving the most stable minimum, $I$, is considered to correspond with that obtained by X-ray analy$\operatorname{sis}^{7,8,10,11}$. Among the other three minima, only the second minimum, II, is considered to be significant since the remaining minima are separated by relatively large energy barriers. On the other hand, the minima of I and II are separated by a barrier of $0.5 \mathrm{kcal} / \mathrm{mol}$ of $\mathrm{CH}_{2}$, which is considered to be small enough for a molecular chain in the normal state I to change

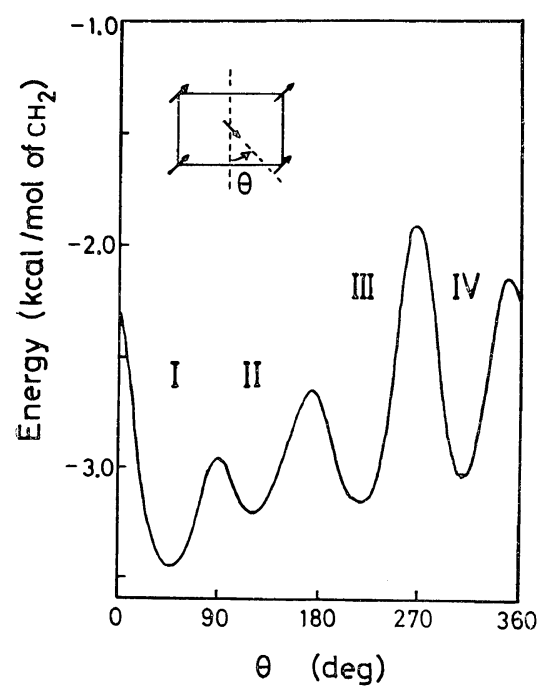

Figure 1. Intermolecular energy for rigid rotation of a polyethylene molecule around its chain axis in a fixed crystallographic environment, calculated by Wada and Hayakawa ${ }^{16}$. to the abnormal secondary state II, especially when it is taken into account that the actual rotation of molecular segments comparable with the lamellar thickness in their length might occur with concurrent motions of smaller parts of the molecular segments, instead of the rotation of whole segments as a rigid rotator. Similar results have been obtained in the calculations by some other authors ${ }^{17,18,24}$, though there are differences in the absolute values of the energy depending on the difference in the selection of potential functions employed in their calculations. For example, McCullough, et al., ${ }^{18}$ evaluated the potential barrier as $0.7 \mathrm{kcal} / \mathrm{mol}$ for the $\mathrm{CH}_{2}$ unit, whereas Oyama, et al., ${ }^{24}$ obtained a value of $0.2 \mathrm{kcal} / \mathrm{mol}$.

As shown in Figure 2, two kinds of molecular chains (1 and 2) pass through one unit-cell of a polyethylene crystal. Normal and abnormal orientations of each polyethylene chain are shown in Figure 3 and are denoted by I and II,

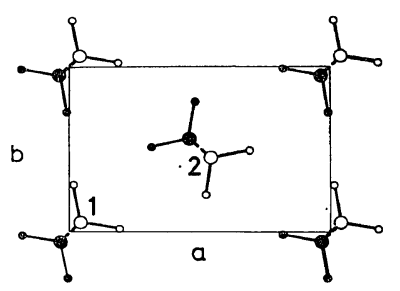

Figure 2. Projection to the $a b$ plane of the crystal structure of polyethylene. Black and white atoms are situated in different planes.

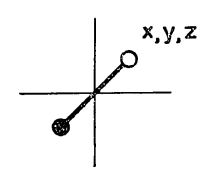

Chain-1 (1)

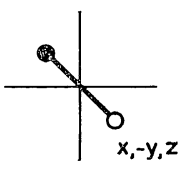

Chain-1 (II)

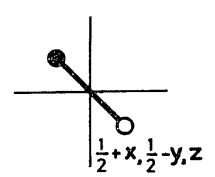

Chain-2 (I)

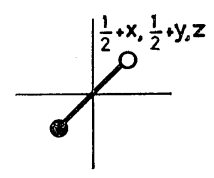

Chain-2 (II)
Figure 3. Normal (I) and abnormal (II) orientations of chain 1 and chain 2 , and fractional coordinates of white carbon atoms. 


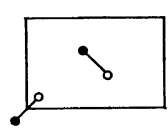

A

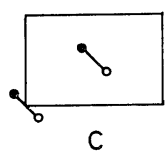

C

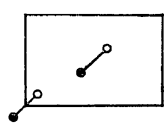

B

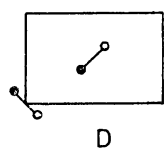

D
Figure 4. Unit-cell structures considering rotational transition of chain-1 and chain-2: (A), normal structure; (B), (C), (D), abnormal structure.

respectively. Depending on the state of the chains of 1 and 2 in Figure 3, four kinds of unit-cell structures are conceivable as shown in Figure 4. Structure $\mathrm{A}$ is the normal structure in which both chains remain in the normal orientation, while the other three structures are the abnormal ones. One of the two chains is in the abnormal orientation in structures $B$ and $\mathrm{C}$, and both of the chains are in the abnormal state in structure $D$. If the probability of a chain being in the normal orientation is denoted by $r$, that of a chain in the abnormal state is $1-r$. Then, the probabilities of existence of $\mathrm{A}$, $\mathrm{B}, \mathrm{C}$, and $\mathrm{D}$ in the crystal are $r^{2}, r(1-r)$, $(1-r) r$ and $(1-r)^{2}$, respectively.

The X-ray intensity of Laue-Bragg reflection, $I_{0}$, for imperfect crystals is written as follows ${ }^{19}$

$$
I_{0}=I_{\mathrm{e}} \bar{F}^{2} G(\mathbf{b})
$$

where $I_{0}, \bar{F}$, and $G(\mathbf{b})$ are the intensity of X-ray scattering by one electron, the average structure factor and Laue function, respectively. By taking account of the four kinds of unit-cell structures and their probabilities of existence, the average structure factor is expressed by eq 2 .

$$
\bar{F}=r^{2} \cdot F_{\mathrm{A}}+r(1-r) \cdot F_{\mathrm{B}}+(1-r) r \cdot F_{\mathrm{C}}+(1-r)^{2} \cdot F_{\mathrm{D}}
$$

where $F_{\mathrm{A}}, F_{\mathrm{B}}, F_{\mathrm{C}}$, and $F_{\mathrm{D}}$ are the structure factors of the unit cells of $\mathrm{A}, \mathrm{B}, \mathrm{C}$, and $\mathrm{D}$, respectively.

The setting angle of the skeletal zigzag plane of polyethylene molecules to the $a$ axis is almost $45^{\circ}$, which permits the representation of the coordinates of each carbon atom in state I and II as indicated in Figure 3. By using these atomic coordinates (fractional coordinates), the structure factors of four unit-cell structures are written by the following equations

$$
\begin{array}{rl}
F_{\mathrm{A}}=2 & f\{\cos 2 \pi(h x+k y+l z) \\
+ & \left.(-1)^{h+k} \cos 2 \pi(h x-k y+l z)\right\} \\
F_{\mathrm{B}}=2 & f\{\cos 2 \pi(h x+k y+l z) \\
+ & \left.+(-1)^{h+k} \cos 2 \pi(h x+k y+l z)\right\} \\
F_{\mathrm{C}}=2 & \{\cos 2 \pi(h x-k y+l z) \\
\left.+(-1)^{h+k} \cos 2 \pi(h x-k y+l z)\right\} \\
F_{\mathrm{D}}=2 f\{\cos 2 \pi(h x-k y+l z) \\
\left.+(-1)^{h+k} \cos 2 \pi(h x+k y+l z)\right\}
\end{array}
$$

where $f$ is the atomic scattering factor of a carbon atom including the temperature factor which is assumed to be isotropic. By substituting eq $3 a-3 d$ into eq 2 , the average structure factor $\bar{F}$ for the $h k l$ reflections with $h+k=$ even is given by

$$
\begin{array}{rl}
\bar{F}=2 & f\{\cos 2 \pi(h x+k y+l z) \\
& \left.+(-1)^{h+k} \cos 2 \pi(h x-k y+l z)\right\}=F_{\mathrm{A}}
\end{array}
$$

and that for the reflections with $h+k=$ odd

$$
\begin{aligned}
\bar{F}= & (2 r-1) 2 f\{\cos 2 \pi(h x+k y+l z) \\
& \left.+(-1)^{h+k} \cos 2 \pi(h x-k y+l z)\right\}=S F_{\mathrm{A}}
\end{aligned}
$$

where $S$ is defined as

$$
S \equiv 2 r-1 \quad(0 \leq S \leq 1)
$$

When $S$ or $r$ is equal to one, the crystal is in a completely ordered state where all polyethylene chains are in a normal orientation. On the other hand, when $S$ is equal to zero or $r$ is equal to one half, the crystal is in a completely disordered state where the probabilities of the chains in state I and II are the same.

Being based on these considerations, the following conclusions are deduced.

(1) Structure factors of $h k l$ reflections with $h+k=$ even are the same to those of the normal structure. Therefore, the transition from I to II has no influence on the intensities of these reflections.

(2) Structure factors of $h k l$ reflections with $h+k=$ odd are proportional to $S$. Therefore, the diffraction intensities corresponding with these reflections are proportional to the square of $S$, decrease with the increasing fraction of 
molecular chains in abnormal orientation and vanish at $S=0$. Consequently, the probability of the rotational transition can be evaluated by inspecting the intensities of these reflections.

$\mathrm{X}$-ray scattering by hydrogen atoms was neglected because of their small scattering power compared to that of carbon atoms. This neglect, however, does not change the final conclusions concerning the relation of the rotational transition of molecular chains to the average structure factor.

It is interesting to compare the above theoretical conclusions with those of the orderdisorder transition in the case of alloy crystals such as $\mathrm{FeAl}$ or $\mathrm{Cu}_{3} \mathrm{Au}^{19}$. The parameter $S$ defined by eq 5 corresponds to the long range order of the lattice of the alloy crystal. The reflections with $h+k=$ odd, which disappear in a completely disordered state, are comparable with the superlattice reflections, while those with $h+k=$ even, which are observable in both of the ordered and disordered state, correspond to the normal reflections in the order-disorder transition.

\section{EXPERIMENTAL}

\section{Sample}

The sample used in this work was a commercial high-density linear polyethylene, Hizex $1200 \mathrm{~J}$, the product of Mitsui Petrochemical Industries Co., Ltd. Its molecular weight $\left(M_{v}\right)$ was 30800 and the degree of branching was 2$\mathrm{CH}_{3} / 1000 \mathrm{CH}_{2}$.

The sample was molten at $180^{\circ} \mathrm{C}$, cooled to room temperature at the rate of $0.1{ }^{\circ} \mathrm{C} / \mathrm{min}$, and annealed at $120^{\circ} \mathrm{C}$. In addition to the bulkcrystallized sample, a single crystal sample was used. Single crystals were prepared from a $0.05-\%$ xylene solution by isothermal crystallization at $60^{\circ} \mathrm{C}$, which were filtered to form an isotropic aggregate. A preferred orientation of crystals was not found in either sample type as proved by the X-ray diffraction method.

\section{$X$-ray Scattering Measurements}

The specimens were attached to the sample holder of the low temperature device of Rigakudenki Company. X-ray intensities of Bragg reflections were measured as a function of temperature by the reflection method by using an X-ray diffractometer with Ni-filtered $\mathrm{Cu}-\mathrm{K}_{\alpha}$ radiation and a Geiger-Müller counter. Scanning of the diffraction intensity was made at the rate of $0.25^{\circ} / \mathrm{min}$. The temperatures of the sample were measured by the $\mathrm{Cu}-$ Constantan thermocouple.

\section{RESULTS}

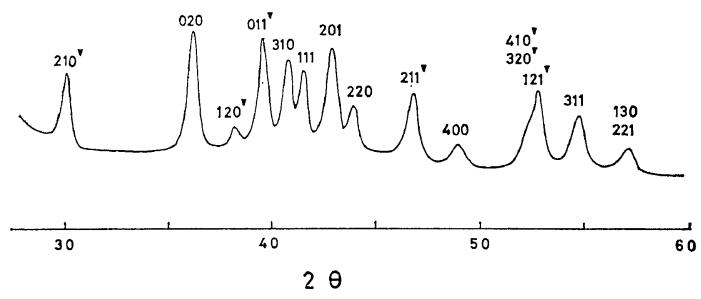

Figure 5. X-ray diffraction spectra at $16^{\circ} \mathrm{C}$ in the range of $27^{\circ} \leq 2 \theta \leq 60^{\circ}$. Reflections with $h+k=$ odd are denoted by prime.

Figure 5 shows X-ray diffraction spectra in the range of diffraction angles $27^{\circ} \leq 2 \theta \leq 60^{\circ}$ at the room temperature for the bulk-crystallized sample. Sixteen diffractions are observed, wherein reflections with $h+k=$ odd are denoted by prime.

In order to evaluate the rotational transition from I to II from the intensities of the reflections with $h+k=$ odd, it is necessary to keep the intensity of X-ray incident beam strictly constant during the measurements, which is operationally difficult. Moreover, the decrease of the diffraction intensities due to the increase of the thermal vibration amplitude, which gives rise to decrease of $f$, should be taken into account. Both of these difficulties are eliminated by adopting the relative values of the integrated intensities of the reflections with $h+k=$ odd to those with $h+k=$ even, that is, $\sum w_{\mathrm{j}} I_{\mathrm{j}}(h+k=$ odd)/ $\sum w_{\mathrm{j}} I_{\mathrm{j}}\left(h+k=\right.$ even), where $I_{\mathrm{j}}$ and $w_{\mathrm{j}}$ are the integrated intensity and the weighting factor of each reflection, respectively. From eq $4 a$ and $4 \mathrm{~b}$, it is predicted that this relative value decreases in proportion to the square of $S$ with proceeding transitions.

The intensities of $210,211,121,410$, and 320 reflections and those of $020,201,220,400$, and 310 reflections were used in the estimation of the relative values in this study. These reflec- 


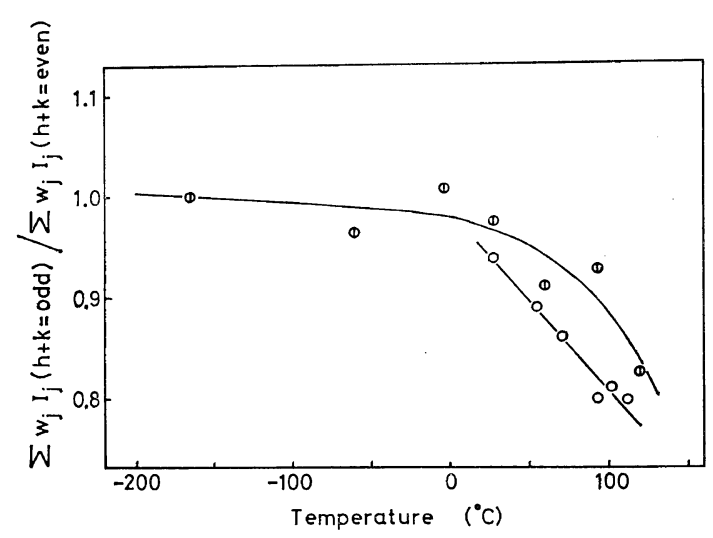

Figure 6. Relative value vs. temperature:

(1), bulk crystal (slowly cooled);

$\bigcirc$, single crystal $\left(T_{x}=60^{\circ} \mathrm{C}\right)$.

tions were selected due to their locations which obviate the superposition of the odd reflections on the even reflections at any temperature. The reciprocal of the standard deviation of each observed intensity was adopted as the weight of each reflection $w_{\mathrm{j}} .{ }^{20}$

Figure 6 shows the temperature dependence of the relative value for the single crystal sample (open circle) and for the bulk-crystallized sample (circle with centerline). The relative value at the lowest temperature $-165^{\circ} \mathrm{C}$ is assumed to be 1.0, and other values are normalized with this value. The standard deviation of each value is about $2 \%$ due to fluctuations of observed intensity data. The relative value is almost constant below $0^{\circ} \mathrm{C}$, but shows a decreasing tendency with increasing temperature above $0^{\circ} \mathrm{C}$. This observation indicates a more rapid decrease in the intensities of the reflections with $h+k=$ odd compared with those of the reflections with $h+k=$ even. This fact suggests that the rotational transition of polyethylene chains is proceeding in the temperature region above $0^{\circ} \mathrm{C}$. If the molecular chains in the crystal of the bulk-crystallized sample are assumed to be in a completely ordered state at $-165^{\circ} \mathrm{C}$, the long range order $S$ at $120^{\circ} \mathrm{C}$ is estimated to be approximately 0.9 , and the molecular chains in the abnormal orientation are about $5 \%$ of all chains in the crystal.

In this estimation, atomic coordinates of $x$ and $y$ are assumed to be constant, being inde- pendent of temperature. This assumption will be discussed in the next section.

\section{DISCUSSION}

In our previous paper ${ }^{7}$ on X-ray analysis of the crystal structure of polyethylene, it was assumed that all molecular chains were in the normal orientation. According to the results, the coordinates of carbon atoms varied slightly with increasing temperature above $0^{\circ} \mathrm{C}$ in accordance with the tendency that the molecular zigzag plane turned toward the $a$ axis. Similar results were also reported by Kasai and Kakudo $^{10}$, and Kavesh and Schultz ${ }^{11}$. It should be noticed that the atomic coordinates observed in these analyses are the averaged ones of the various atomic positions which are actually different with each other depending on the different local structure caused by crystal defects. When some of the molecular chains in the normal orientation transform to the abnormal orientation, the atomic coordinates might be observed on an average by the conventional analysis indicating that the molecular zigzag plane turns toward the $a$ axis.

Thus, the following two models of the molecular motions are conceivable. The first is the model in which all polyethylene chains uniformly turn slightly toward the $a$ axis, and the second is the model wherein a portion of the molecular chains rotates by an angle of about $90^{\circ}$ from their normal orientation to give the abnormal orientation. The decision between such models might be possible, if the X-ray analysis is made by considering the degree of the rotational transition, existence of other crystal defects, and, at the same time, variations of atomic coordinates and auisotropic temperature factors of both carbon and hydrogen atoms. Such an analysis requires too many parameters compared with the number of available X-ray diffraction data. In spite of these difficulties, we would like to adopt the latter model related to the rotational transition based on the following consideration.

The viscoelastic measurements of polyethylene single crystals ${ }^{1,2}$ show the absorption in their mechanical relaxation curves in the temperature region between $0^{\circ} \mathrm{C}$ and the melting temperature. 
While the absorption maximum is located at about $80-90^{\circ} \mathrm{C}$ at $110 \mathrm{~Hz}$, it shifts to $15^{\circ} \mathrm{C}$ at $10^{-4} \mathrm{~Hz}$ according to the transition map prepared by the conversion of stress-relaxation data to the dynamic complex modulus ${ }^{25}$. Such a crystalline absorption is considered to be due to the internal frictions caused by rubbing of neighboring molecules in thermal motions in the crystal lattice. The rotational transition of molecular chains treated in this study is considered to be one of the important mechanisms associated with the viscoelastic crystalline absorption.

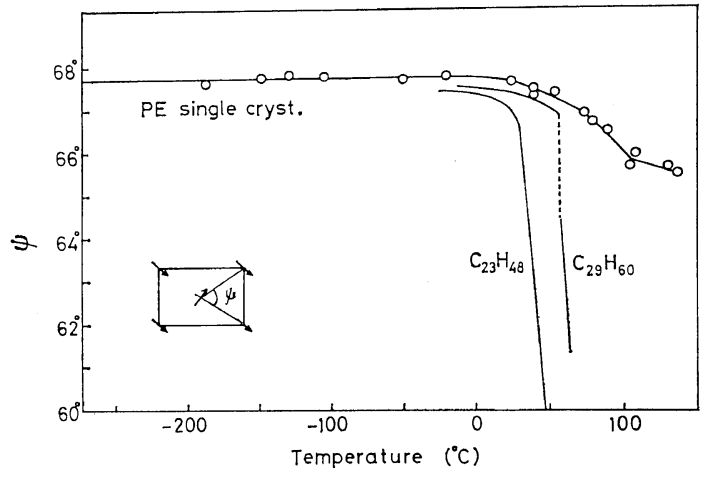

Figure 7. Angle $\psi$ vs. temperature for a polyethylene single crystal prepared at $50^{\circ} \mathrm{C}$ (this work), and $n$-paraffin (Müller ${ }^{23}$ ).

Our previous measurements ${ }^{5}$ show that the unit cell of polyethylene crystal shows an anisotropic thermal expansion and approaches the hexagonal form to some extent at the temperatures above $0^{\circ} \mathrm{C}$. Figure 7 shows the temperature dependence of the angle between both diagonals of the $a b$ plane of the unit cell, $\psi$, for polyethylene single crystals and normal paraffins. Crystal is in a hexagonal form when $\psi$ is equal to $60^{\circ}$. The result that the value of $\psi$ decreases above $0^{\circ} \mathrm{C}$ toward $60^{\circ}$ is considered to be consistent with the concept of the rotational transition of molecular chains in this temperature region, because the statistical rotational transition of the molecular zigzag plane gives more or less radial symmetry around the molecular axis.

The unit-cell volume and the disorder factor of polyethylene single crystals observed at room temperatures were increased by annealing at $90^{\circ} \mathrm{C}$ or $100^{\circ} \mathrm{C}^{21}$. This observation will be explained by assuming that the abnormal structures are increased at the annealing temperature and retained in the crystal after cooling to room temperature.

It appears unlikely that a molecular segment corresponding to the lamellar height, which contains 80 or more $\mathrm{CH}_{2}$ units, rotates as a rigid rotator and transforms to the abnormal orientation, because it requires a passage over a barrier of $40 \mathrm{kcal} / \mathrm{mole}$ of segment to achieve the transition ${ }^{16}$. Actual mechanism will be like that the rotational transition proceeds by the motion of the smaller part of segment corresponding with various modes of torsional motion of the molecular chain. This mechanism will be supported by the fact that the reduction of the fiber period of polyethylene molecule is fairly large above room temperature ${ }^{22}$.

The abnormal orientation of chains, which is regarded as one kind of defect in the crystal, gives rise to an increase of entropy and consequently a decrease of free energy of the crystal at high temperatures.

The long range order $S$ is relatively large even at the temperatures just below the melting point. For example, $S$ is 0.9 at $120^{\circ} \mathrm{C}$ as already estimated. If the melting of the crystal is entirely related to the rotational transition of molecules, $S$ is expected to decrease to zero at the melting temperature. Thus, in order to explain the melting of the crystal, it is necessary

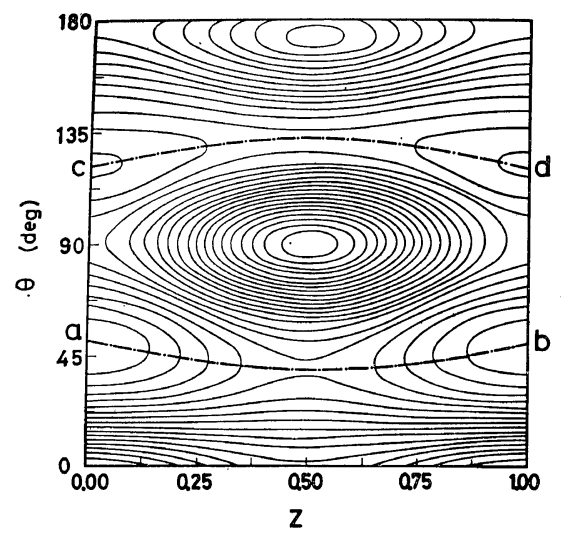

Figure 8. Energy contour map for rotation, $\theta$, around molecular axis vs. translation, $Z$, along molecular axis. Contour lines are $0.1 \mathrm{kcal} / \mathrm{mol}$ of $\mathrm{CH}_{2}$ (McCullough and Lindenmeyer $\left.{ }^{18}\right)$. 


\section{K. IOHARA, K. ImADA, and M. TAKayAnAGI}

to take into account other kinds of molecular motions in addition to the rotational transition.

Concerning this subject, McCullough and Lindenmeyer ${ }^{18}$ have recently shown the possibility of the translation of a molecule along the chain axis in addition to the rotation around the same axis based on an energetical calculation. Their result is shown in Figure 8, where $a$ and $b$ represent the normal orientation $I$ and $\mathrm{c}$ and $\mathrm{d}$ correspond to the abnormal orientation II in our paper. They reported that a polyethylene chain can translate along the chain axis by one repeat unit energetically more easily through the pass of $a c d b$ than the pass of $a b$ especially at high temperatures. The unit-cell structures before and after translation of one repeat unit are the same. Therefore, it is impossible to evaluate the probability of the translation from $a$ to $b$ and that from $c$ to $d$ by using the similar procedure applied to the rotational transition in this study. However, the existence of molecular chains in the abnormal orientations, $c$ or $d$, found in our study is not considered to deny the mechanism of the translation through the pass of acdb, but rather to partially support the prediction by McCullough and Lindenmeyer ${ }^{18}$.

Acknowledgement. The authors express their sincere thanks to Dr. Oyama in Department of Applied Science of Kyushu University for his useful discussions and Mr. Suehiro of this laboratory for his helpful technical advice.

\section{REFERENCES}

1. M. Takayanagi and T. Matsuo, J. Macromol. Sci., B1, 407 (1967).

2. S. Manabe, A. Sakoda, A. Katada, and M. Takayanagi, ibid., B4, 161 (1970).

3. A. Odajima, A.E. Woodward, and J.A. Sauer, J. Phys. Chem. 66, 718 (1962).

4. S. Iwayanagi and I. Miura, J. Appl. Phys. Japan, 4, 94 (1965).
5. K. Iohara, K. Imada, and M. Takayanagi, Rep. Progr. Polym. Phys. Japan, 12, 183 (1969).

6. Y. Aoki, A. Chiba, and M. Kaneko, J. Phys. Soc. Japan, 27, 1579 (1969).

7. K. Iohara, K. Imada, and M. Takayanagi, Polymer J., 3, 357 (1972).

8. C.W. Bunn, Trans. Faraday Soc., 35, 482 (1939).

9. H.G. Kilian, Kolloid-Z. Z. Polym., 183, 1 (1962): 185, 13 (1962).

10. N. Kasai and M. Kakudo, Rep. Progr. Polym. Phys. Japan, 11, 145 (1968).

11. S. Kavesh and J.M. Schultz, J. Polym. Sci., Part A-2, 8, 243 (1970).

12. T. Kitagawa and T. Miyazawa, Rep. Progr. Polym. Phys. Japan, 11, 219 (1968).

13. A. Peterlin and E. W. Fischer, Z. Phys., 159, 272 (1961).

14. A. Peterlin, E.W. Fischer, and Chr. Reinhold, J. Chem. Phys., 37, 1403 (1962).

15. K. Okano, Rikagaku Kenkyūsho Hōkoku (Rept. Inst. Phys. Chem. Res.), 40, 295 (1964).

16. Y. Wada and R. Hayakawa in "Progress in Polymer Science, Japan," Vol. 3, S. Okamura and M. Takayanagi, Ed., Kodansha, Tokyo, 1972, p 215.

17. P.E. McMahon, R. L. McCullough, and A. A. Schlegel, J. Appl. Phys., 38, 4123 (1967).

18. R. L. McCullough and P. H. Lindenmeyer, General Lecture presented at Int. Conf. Mech. Behavior of Materials, Kyoto, Aug. 1971.

19. "X-sen Kesshōgaku (X-ray Crystallography)," Vol. II, Y. Nitta, Ed., Maruzen Co., Ltd., Tokyo, 1961, Chapter 4.

20. ibid., Chapter 3.

21. K. Iohara, K. Imada, and M. Takayanagi, Polymer $J$., in press.

22. K. Iohara, K. Imada, and M. Takayanagi, unpublished work.

23. A. Müller, Proc. Roy. Soc., A127, 417 (1930); A138, 514 (1932).

24. T. Oyama, presented at Microsymposium on Crystal Defect of Polymer Crystal, Fukuoka, Feb. 1972.

25. M. Takayanagi, Kogyo Kagaku Zasshi (J. Chem. Soc. Japan, Ind. Chem. Sect.), 73, 1277 (1970). 